\title{
JOVENES, VALORES Y UTILIZACIÓN DE LA TECNOLOGÍA EN EL TIEMPO DE OCIO
}

\author{
Jovens, valores e uso da tecnologia no tempo de lazer \\ Youth, values and use of technology in leisure time
}

\author{
Javier Páez Gallego \\ Universidad Nacional de Educación a Distancia (UNED) \\ Angel De-Juanas Oliva \\ Universidad Nacional de Educación a Distancia (UNED) \\ Ángel Luis Gonzáles Olivares \\ Profesor Asociado do Departamento de Pedagogia. \\ Universidad de Castilha La Mancha \\ ALuis.Gonzalez@uclm.es
}

\section{Resumen}

Las pautas de utilización de las tecnologías de la comunicación y la información en el tiempo de ocio de los jóvenes están relacionadas con su sistema de valores. El presente trabajo analiza la relación entre ambas variables. Han participado 385 jóvenes con edades comprendidas entre los 13 y los 19 años. Todos ellos respondieron por su preferencia de utilización de nuevas tecnologías y su escala axiológica. Se concluye la relación significativa entre los valores de los jóvenes y la utilización de la tecnología en el tiempo de ocio; así se concluyen diferencias entre ambas variables en función de la variable sexo en el caso de los videojuegos. Se discuten complicaciones educativas y se señalan líneas de continuidad en nuestro estudio.

Palabras clave: Valores. Tecnología de la información. Ocio.

\section{Resumo}

Os padrões de uso de tecnologias de comunicação e informação em tempo de lazer dos jovens estão relacionados ao seu sistema de valores. Este artigo discute a relação entre duas variáveis. Eles têm 385 jovens com idades entre 13 e 19 anos participaram. Eles responderam preferência pela utilização de novas tecnologias e sua escala axiológica. A relação significativa entre os valores dos jovens e o uso da tecnologia no tempo de lazer está concluído; e as diferenças entre as duas variáveis com base na variável sexo, no caso de jogos de vídeo são concluídos. Complicações educacionais são discutidos e linhas de continuidade identificados em nosso estudo. 
PROGRAMA DE PÓS-GRADUAÇÃO EM COMUNICAÇÃO DA UNIVERSIDADE FEDERAL DE SANTA MARIA

Palavras-chave: Valores. Tecnologia da informação. Lazer.

\begin{abstract}
The patterns of use of communication technologies and information in the leisure time of young people are related to their value system. The present work analyzes the relationship between both variables. There have been 385 young people aged between 13 and 19. All of them responded by their preference of using new technologies and their axiological scale. It concludes the significant relationship between the values of young people and the use of technology in leisure time; Thus concluding differences between both variables as a function of the variable sex in the case of video games. Educational complications are discussed and lines of continuity are indicated in our study.
\end{abstract}

Key words: Values. Information technology. Leisure.

\title{
1. INTRODUCCIÓN
}

La realización de actividades de ocio tiene una gran importancia para el desarrollo humano y se encuentra mediatizada por las decisiones de cada persona; y éstas, a su vez, por los valores que la identifican (Ferreira, Pose, \& De Valenzuela, 2015). Así, el desempeño de actividades de ocio de corte social y altruista, como por ejemplo todas las relacionadas con los espacios en los que pueden tener mayor percepción de libertad y menor monitorización, puede estar relacionado con valores prosociales y de búsqueda del bien común (Ortega, Lazcano \& Rocha, 2015). Por el contrario, las actividades de ocio relacionadas con el disfrute individual tienen un relación estrecha con valores de autosatisfacción (Corvalán, 2012).

En un sentido similar, el uso de las tecnologías en el tiempo de ocio está vinculado con el sistema axiológico de los individuos que las utilizan (Matza \& Sykes, 2015; Lujambio, Martínez, \& Samaniego, 2017). Este vínculo toma una importancia capital durante la adolescencia, especialmente: cuando se produce un incremento en la percepción de la autonomía personal; el grupo de iguales adquiere un papel primordial en el proceso de socialización; y, la alfabetización tecnológica ocurre, fundamentalmente, en contextos de educación informal (Medrano \& Aierbe, 2008; Medrano, Aierbe \& Martínez, 2011; Fernández-García, Poza-Vilches \& Fiorucci, 2015; Wieckowski \& White, 2017).

\section{MARCO TEÓRICO}

En los últimos años, la utilización de la tecnología ha tenido un papel preponderante en las actividades de ocio de los adolescentes ([Autor/a]; Melendro, García-Castilla \& Goig, 2016;). Así, el uso de los avances informáticos y digitales es destacado en todos sus formatos 
PROGRAMA DE PÓS-GRADUAÇÃO EM COMUNICAÇÃO DA UNIVERSIDADE FEDERAL DE SANTA MARIA

y soportes; de tal manera que más del $98.9 \%$ de los jóvenes españoles recurren a ellos en sus ratos de ocio según el Informe de ocio y tecnología del INJUVE (2015).

Por otro lado, las tecnologías de ocio siempre han tenido un papel fundamentalmente mediador de las relaciones sociales de los jóvenes (Loader, Vromen, \& Xenos, 2014). Al respecto, Solano, González \& López (2013), consideran que el uso de la tecnología se ha convertido en un medio para fomentar las relaciones sociales entre los jóvenes. Si bien, los avances actuales han contribuido a superar el papel mediador de las tecnologías y, recientemente, el uso de la tecnología se ha convertido en un fin en sí mismo, por el cual los adolescentes se relacionan influidos por un elemento tecnológico sin necesidad de contacto e interacción directa. De tal manera se provoca una dependencia directa de la tecnología para favorecer las relaciones sociales y potenciar la aparición e incremento de trastornos de dependencia de los jóvenes a los medios digitales (Rosen, Whaling, Carrier, Cheever, \& Rokkum, 2013; González, Fernández, \& Urturi, 2015; Zambrano \& Badillo, 2016). Numerosos estudios arrojan datos transversales de la evolución del uso de las tecnologías en el tiempo de ocio de los jóvenes mostrando resultados similares. De tal modo, actividades como ver la televisión, oír la radio, usar el ordenador, el móvil o jugar con videojuegos son actividades recurrentes que los jóvenes realizan en su tiempo de ocio (Van Quaquebeke, Graf, Kerschreiter, Schuh, \& Dick, 2014; Martínez, 2015; Elizalde, 2016).

Por otra parte, durante la juventud tiene lugar la adolescencia. Este periodo puede ser considerado como una etapa de definición y estabilización inicial de la personalidad de los jóvenes. Así, dimensiones como el sistema de valores alcanzan un carácter propio definido durante esta etapa evolutiva y es un momento fundamental para estudiar su estructura y contenido concreto (Paez, 2014; García, Barbero, \& Muñoz, 2017).

Además, teniendo en cuenta la concreción de los valores en actitudes y éstos en conductas, el estudio de la dimensión axiológica permite conocer la motivación de las decisiones. De esta manera, el modelo de valores universales de Schwartz permite operativizar el estudio de los valores representado en un sentido circular. Según esta propuesta, los valores, en su dimensión psicológica, son conceptualizados como constructos vinculados a las emociones cuya función es guiar las decisiones y los juicios. Estos valores pueden ser, además, ordenados por orden de importancia o prioridad, conformando el sistema axiológico propio de cada individuo (Torres, Schwartz, \& Nascimento, 2016).

El modelo de Schwartz está formado por diez valores de carácter universal, en el cual cada valor se encuentra próximo entre sí a aquellos axiomas cuya identificación y logro se 
relaciona y resulta congruente. Por el contrario, cada valor se representa enfrentado y de manera opuesta con aquel valor cuya consecución simultánea es incompatible.

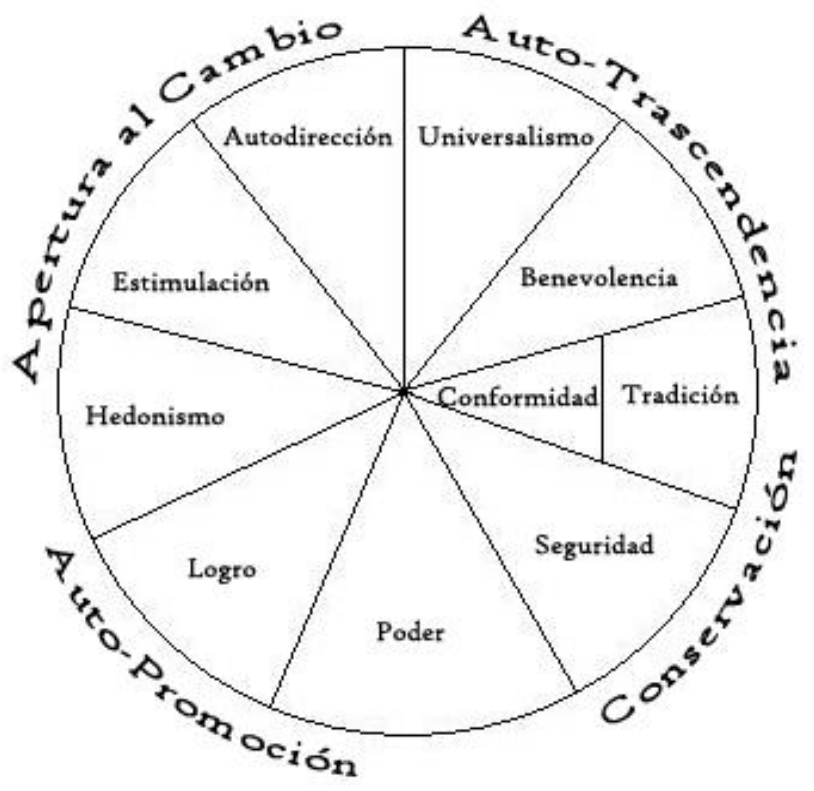

Figura 1. Modelo de Schwartz de las relaciones entre valores.

Fuente: Lindeman \& Verkasalo (2005)

El contenido motivacional de los diez valores es el siguiente: autodirección: responde a la necesidad individual de control del medio y de interacción autónoma e independiente; estimulación: es la necesidad de mantener un nivel óptimo de activación y la obtención de vivencias de alta intensidad; hedonismo: tiene que ver con las necesidades orgánicas y de placer asociadas a una satisfacción ineludible; seguridad: son las exigencias básicas de supervivencia individual y grupal; conformidad: supone la inhibición de inclinaciones individuales potencialmente disruptivas para el funcionamiento armónico del grupo, priorizando así las necesidades colectivas sobre los deseos individuales; tradición: es la expresión de la solidaridad y la singularidad grupal; logro: implica tener competencia para obtener recursos y aprobación social; poder: hace alusión a la necesidad individual de dominio y control; benevolencia: es la necesidad de interacción positiva para promover el bien del grupo. Relacionado con la necesidad de afiliación; universalismo: se trata de las necesidades de supervivencia de sujetos y grupos cuando los recursos de los que depende la 
vida son escasos y compartidos.

A su vez, estos diez valores se ordenan en cuatro grandes valores de orden superior contrapuestos entre sí dos a dos (Schwartz, 1992; Musitu, 2000), a saber: apertura al cambio vs. conservación: se agrupan valores de estimulación y auto-dirección y, por otro lado, valores de seguridad, conformidad y tradición; y, auto-beneficio vs. autotrascendencia: estas dimensiones agrupan los valores de poder y logro, enfrentándose a los de universalismo y benevolencia.

Considerando así el uso de las tecnologías de la comunicación y la información, así como y dimensión axiológica de los jóvenes, numerosos trabajos han tratado de arrojar evidencias sobre su vinculación. Así, la investigación llevada a cabo por Amorós, Buxarrais y Casas (2002) estudió la relación de las TIC con los procesos perceptivos, comunicativos y axiológicos de los adolescentes de 12 a 16 años, encontrando una relación positiva y directa entre las variables.

Unos años más tarde, en un estudio similar, Delgado, Arrieta y Riveros (2009), hallaron una relación entre el uso didáctico de las TIC en contextos educativos y el desarrollo de responsabilidades y valores positivos en los educandos, ofreciendo un programa de seis talleres que, mediante las nuevas tecnologías, promovía estos valores.

Otros estudios, también han encontrado resultados parejos en cuanto a una relación significativa entre el uso de las tecnologías de la información y la comunicación (en adelante, TIC) y el sistema axiológico de los jóvenes. Así, los trabajos de Samaniego y Cortés (2009) y Montero (2013) encontraron relación entre los contenidos preferidos por los jóvenes en el consumo de TIC y el sistema de valores que se adquiere, produciéndose procesos de asimilación axiológica y, por ende, desarrollando actitudes y conductas coherentes con estos valores y semejantes a los observados en los medios de comunicación de masas.

Por último, González, Fernández y Urturi (2015) y Del Barrio, Martínez (2016) han analizado el uso de las últimas tecnologías en el tiempo de ocio de los jóvenes. De tal modo, han determinado que el uso de los medios tecnológicos resultan ser una actividad preferente dentro del tiempo de ocio de los jóvenes con edades comprendidas entre 12 y 18 años. Además, considerando la relación entre los contenidos preferidos y los valores, el sistema axiológico de los jóvenes tiene una doble vinculación: por un lado, como tendencia a la selección de este tipo de actividad de ocio; y, por otro lado, como variable influenciada por los contenidos consumidos.

Con todo, a la luz de los estudios precedentes, cabe preguntarse por una mayor 
PROGRAMA DE PÓS-GRADUAÇÃO EM COMUNICAÇÃO DA UNIVERSIDADE FEDERAL DE SANTA MARIA

profundización en el estudio del vínculo existente entre los valores de los jóvenes y el uso de las TIC. Por ello, el presente trabajo tiene como principal objetivo analizar la relación existente entre el sistema de valores de los jóvenes y la utilización de TIC durante su tiempo de ocio. Además, se pretende determinar cuáles son las tecnologías más utilizadas en función de los valores que definen el sistema axiológico y la influencia de la variable sexo y edad en la variabilidad en las elecciones.

\section{METODOLOGÍA}

Teniendo en cuenta el objetivo planteado se establece un diseño de investigación caracterizado por ser cuantitativo y no experimental, así como descriptivo de tipo ex-post facto, dado que no se aplicaron tratamientos previa ni posteriormente a la recogida de los datos. Es, además, transversal y correlacional en el que se estudian las relaciones existentes entre las variables seleccionadas.

\section{Participantes}

En el estudio han participado un total de 385 alumnos de Educación Secundaria Obligatoria y Bachillerato de centros públicos, privados y concertados de la Comunidad Autónoma de Madrid (España) (hombres, $n=191$, 49.6\%; mujeres, $n=194,50.4 \%$ ) con edades comprendidas entre los 13 y los 19 años.

La muestra, con una edad media cercana a los 16 años $(M=15.47 ; D T=1.56)$, se seleccionó mediante un mиеstreo inicial de carácter intencional del que se obtuvo una muestra inicial total de 1262 estudiantes. Posteriormente, se llevaron a cabo afijaciones proporcionales en función de estratos en los que se tuvo en cuenta: el tamaño de la población de estudiantes de la Comunidad de Madrid, el tipo de estudio, la titularidad de centro, el sexo y el curso. El cálculo del tamaño muestral final, según fórmula, reveló que el volumen mínimo de la muestra representativa debía ser de 385.

\section{Procedimiento}

La aplicación se realizó mediante prueba de papel y lápiz y de manera colectiva en los centros educativos durante el horario lectivo. La duración de la aplicación fue en una única sesión de aproximadamente cuarenta y cinco minutos. Se siguieron los principios éticos de la Declaración de Helsinki (2013); se informó a los participantes de los propósitos del estudio, la 
participación fue voluntaria, anónima y bajo instrucciones pautadas. Se aseguró la confidencialidad de las respuestas y se evitó el sesgo del aplicador. El trabajo de campo se llevó a cabo durante los años 2015 y 2016.

\section{Instrumentos}

Para la recogida de los datos se empleó un cuestionario elaborado ad hoc sobre la preferencia de uso de los recursos tecnológicos en el tiempo libre de los jóvenes. En dicho cuestionario, los participantes debían responder si utilizaban o no la televisión, la radio, el ordenador personal, videojuegos y dispositivos musicales en su tiempo libre.

Igualmente, se aplicó la Escala de Valores Universales de Schwartz. Este instrumento recoge información sobre el sistema de valores de los participantes y está compuesto por 45 ítems agrupados en 10 factores y éstos, a su vez, se organizan en cuatro valores de orden superior. A su vez, presenta un sistema de respuesta aditiva global tipo Likert en la que todos los ítems están formulados en positivo. Así, las puntuaciones de la escala tienen nueve opciones de respuesta que oscilan entre -1 y 7 . De este modo, las puntuaciones más altas indican, según la subescala, una mayor preferencia de los valores representados en el sistema axiológico de los participantes. Por el contrario, las puntuaciones más bajas muestran una menor preferencia o centralidad con los valores que expresa cada ítem ([Autor/a]).

\section{RESULTADOS}

Una vez creada la base de datos, se planificaron y realizaron análisis descriptivos para representar estadísticamente la muestra. Para analizar la influencia de la utilización de tecnologías en relación al sistema de valores de los jóvenes se realizaron análisis de inferencias mediante el estadístico $t$ de student. Por otro lado, con el objeto de examinar la relación entre la utilización de tecnología y el sexo de los participantes se realizó un análisis de los diferentes ítems mediante Tablas de contingencia, el valor de Chi-cuadrado de Pearson y su significación, así como el coeficiente de correlación Phi y la $V$ de Cramer. Para ello, se utilizó el programa informático SPSS 22.0 para Macintosh. El nivel de confianza establecido fue del $95 \%(\mathrm{p}<.05)$ 
Tabla 1. Medias (M), desviaciones típicas (DT), resultados de la prueba de contrastes de medias y significación con respecto a los valores, jugar a los videojuegos y utilizar el PC.

\begin{tabular}{|c|c|c|c|c|c|c|c|c|c|c|c|c|c|c|}
\hline & \multicolumn{2}{|l|}{ Total } & \multicolumn{2}{|c|}{$\begin{array}{l}\text { Juegan } \\
\text { videojuegos } \\
(\mathrm{N}=157)\end{array}$} & \multicolumn{2}{|c|}{$\begin{array}{l}\text { No juegan a } \\
\text { videojuegos } \\
(\mathrm{N}=\mathbf{2 2 4})\end{array}$} & \multicolumn{4}{|c|}{$\begin{array}{l}\text { Utilizan PC } \\
(\mathbf{N}=319)\end{array}$} & \multicolumn{2}{|c|}{$\begin{array}{l}\text { No utilizan PC } \\
(\mathrm{N}=62)\end{array}$} & \multirow[b]{2}{*}{ Pru. t } & \multirow[b]{2}{*}{$\begin{array}{l}\text { Sig. } \\
\text { (bilat.) }\end{array}$} \\
\hline & $\mathbf{M}$ & DT & $\mathbf{M}$ & DT & $\mathbf{M}$ & DT & Pru. t & $\begin{array}{l}\text { Sig. } \\
\text { (bilat.) }\end{array}$ & M & DT & $\mathbf{M}$ & DT & & \\
\hline Benevolencia & 7.236 & 1.022 & 7.169 & 1.017 & 7.286 & 1.028 & -1.100 & .272 & 7.195 & 1.021 & 7.461 & 1.012 & -1.881 & .061 \\
\hline Seguridad & 7.024 & .968 & 6.994 & .983 & 7.03 & .961 & -.431 & .667 & 6.975 & .998 & 7.254 & .774 & -2.088 & $.037 *$ \\
\hline Universalismo & 6.623 & 1.071 & 6.512 & .992 & 6.714 & 1.103 & -1.830 & .068 & 6.608 & 1.088 & 6.953 & 1.006 & -.946 & .345 \\
\hline Conformidad & 7.116 & 1.160 & 7.025 & 1.144 & 7.186 & 1.169 & -1.339 & .182 & 7.082 & 1.152 & 7.310 & 1.187 & -1.417 & .157 \\
\hline Tradición & 5.819 & 1.086 & 5.768 & 1.099 & 5.858 & 1.068 & -.798 & .425 & 5.791 & 1.070 & 5.974 & 1.131 & -1.220 & .223 \\
\hline Logro & 6.786 & 1.146 & 6.847 & 1.203 & 6.751 & 1.086 & .807 & .420 & 6.794 & 1.075 & 6.770 & 1.416 & .155 & .877 \\
\hline Poder & 5.103 & 1.388 & 5.362 & 1.454 & 4.908 & 1.268 & 3.249 & $.001^{*}$ & 5.097 & 1.361 & 5.089 & 1.361 & .043 & .966 \\
\hline Hedonismo & 7.148 & 1.285 & 7.322 & 1.264 & 7.025 & 1.294 & 2.240 & $.026^{*}$ & 7.126 & 1.288 & 7.258 & 1.292 & -.735 & .463 \\
\hline Estimulación & 6.480 & 1.388 & 6.671 & 1.440 & 6.348 & 1.340 & 2.246 & $.025^{*}$ & 6.461 & 1.384 & 6.586 & 1.426 & -.645 & .519 \\
\hline Autodirección & 7.217 & .930 & 7.240 & .941 & 7.200 & .928 & .411 & .682 & 7.210 & .930 & 7.256 & .948 & -.351 & .726 \\
\hline
\end{tabular}

Fuente: Elaboración propia. ${ }^{*} \rho<.05$. Se han asumido varianzas iguales. 
PROGRAMA DE PÓS-GRADUAÇÃO EM COMUNICAÇÃO DA UNIVERSIDADE FEDERAL DE SANTA MARIA

En la Tabla 1 se observan las diferencias de preferencia de uso de los juegos de videoconsola y PC de los jóvenes en función del perfil axiológico que los define. En el caso del uso de la videoconsola, haciendo un análisis de comparación de medias, encontramos que sólo los jóvenes con un perfil axiológico identificado con los valores poder, estimulación y autodirección, destacan por su preferencia de este recurso tecnológico en su tiempo de ocio (Poder $\mathrm{t}=3.25 ; \mathrm{p}=.001 ;$ Hedonismo t=2.24; $\mathrm{p}=.026$; Estimulación $\mathrm{t}=2.24 ; \mathrm{p}=.005$ ).

Los valores poder, hedonismo y estimulación se relacionan entre sí en la representación gráfica del modelo de valores de Schwartz; lo que permite identificar un perfil axiológico concreto que muestra preferencia por el uso de la videoconsola como actividad de entretenimiento en su tiempo libre. Además, los valores hedonismo y estimulación pertenecen al valor de orden superior Apertura al cambio, que define a personas proclives a asumir riesgos y afrontar situaciones y decisiones novedosas.

Por otro lado, el análisis de la preferencia de uso del PC en el tiempo libre evidencia que únicamente destacan los jóvenes identificados por el valor seguridad. No obstante, el resultado del contraste de medias muestra que el uso que hacen de este recurso tecnológico como actividad de ocio es menor al de los jóvenes identificados con el resto de valores, obteniendo una $\mathrm{t}=-2.088$ y una probabilidad menor a $.05(\mathrm{p}=.037)$ 
PROGRAMA DE PÓS-GRADUAÇÃO EM COMUNICAÇÃO DA UNIVERSIDADE FEDERAL DE SANTA MARIA

Tabla 2. Medias (M), desviaciones típicas (DT), resultados de la prueba de contrastes de medias y significación con respecto a los valores, escuchar la radio y ver TV.

\begin{tabular}{|c|c|c|c|c|c|c|c|c|c|c|c|c|c|c|}
\hline & \multicolumn{2}{|l|}{ Total } & \multicolumn{2}{|l|}{$\begin{array}{l}\text { Escuchan } \\
\text { radio } \\
(\mathrm{N}=200) \\
\end{array}$} & \multicolumn{2}{|c|}{$\begin{array}{l}\text { No escuchan } \\
\text { la radio } \\
(\mathrm{N}=\mathbf{1 8 0})\end{array}$} & \multirow[b]{2}{*}{ Pru. $\mathbf{t}$} & \multirow[b]{2}{*}{$\begin{array}{l}\begin{array}{l}\text { Sig. } \\
\text { (bilat.) }\end{array} \\
\text { (a) }\end{array}$} & \multicolumn{2}{|c|}{$\begin{array}{l}\text { Ven la TV } \\
(\mathrm{N}=324)\end{array}$} & \multicolumn{2}{|c|}{$\begin{array}{l}\text { No ven la TV } \\
(\mathrm{N}=57)\end{array}$} & \multirow[b]{2}{*}{ Pru. t } & \multirow[b]{2}{*}{$\begin{array}{l}\text { Sig. } \\
\text { (bilat. }\end{array}$} \\
\hline & $\mathbf{M}$ & DT & M & DT & $\mathbf{M}$ & DT & & & $\mathbf{M}$ & DT & $\mathbf{M}$ & DT & & \\
\hline Benevolencia & 7.236 & 1.022 & 7.240 & 1.043 & 7.234 & 1.007 & .072 & .943 & 7.218 & 1.038 & 7.389 & .933 & -1.210 & .227 \\
\hline Seguridad & 7.024 & .968 & 7.129 & .935 & 6.893 & .993 & 2.378 & $.018^{*}$ & 7.029 & .054 & 6.968 & .118 & .438 & .661 \\
\hline Universalismo & 6.623 & 1.071 & 6.674 & 1.127 & 6.578 & .073 & .874 & .383 & 6.618 & 1.100 & 6.706 & .820 & -.577 & .564 \\
\hline Conformidad & 7.116 & 1.160 & 7.234 & 1.114 & 6.989 & 1.199 & 2.066 & $.040 *$ & 7.130 & 1.148 & 7.061 & 1.229 & .412 & .681 \\
\hline Tradición & 5.819 & 1.086 & 5.870 & 1.097 & 5.736 & 1.062 & 1.453 & .147 & 5.830 & 1.067 & 5.765 & 1.153 & .424 & .672 \\
\hline Logro & 6.786 & 1.146 & 6.791 & 1.107 & 6.798 & 1.171 & .032 & .974 & 6.756 & 1.145 & 6.687 & 1.068 & -1.416 & .158 \\
\hline Poder & 5.103 & 1.388 & 5.169 & 1.372 & 5.019 & 1.358 & .585 & .287 & 5.103 & 1.396 & 5.052 & 1.175 & .257 & .797 \\
\hline Hedonismo & 7.148 & 1.285 & 7.122 & 1.284 & 7.171 & 1.297 & .839 & .713 & 7.157 & 1.300 & 7.097 & 1.226 & .326 & .744 \\
\hline Estimulación & 6.480 & 1.388 & 6.573 & 1.364 & 6.368 & 1.408 & .912 & .150 & 6.483 & 1.402 & 6.474 & 1.328 & .047 & .963 \\
\hline Autodirección & 7.217 & .930 & 7.243 & .909 & 7.184 & .958 & .569 & .541 & 7.167 & .947 & 7.505 & .787 & -2.548 & $.011 *$ \\
\hline
\end{tabular}

Fuente: Elaboración propia. ${ }^{*} \rho<.05$. Se han asumido varianzas iguales 
De igual modo, en la Tabla 2 se muestran las diferencias en las preferencias de uso de la radio y la televisión en el tiempo de ocio de los jóvenes en función de sus valores. De tal manera, los jóvenes que se definen por los valores seguridad $(\mathrm{t}=-2.378 ; \mathrm{p}=.018)$ y conformidad ( $\mathrm{t}=2.066 ; \mathrm{p}=.040$ ) escuchan la radio en su tiempo de ocio en mayor medida que los jóvenes identificados por cualquiera de los otros valores del modelo de Schwartz.

Los valores de seguridad y conformidad se encuentran situados de manera contigua en la representación del modelo de valores universales (véase Figura 1), por lo que la consecución pareja de los mismos es posible y compatible. Esto permite identificar un perfil axiológico concreto que define a los jóvenes caracterizados por valores y actitudes conservadoras como tendentes a usar la radio como actividad de ocio, destacando por encima de la media de su grupo de edad evolutiva.

Por otro lado, en el uso de la televisión con una función lúdica, sólo los jóvenes identificados con el valor autodirección obtienen una puntuación negativa estadísticamente significativa en el contraste de medias ( $\mathrm{t}=-2.548 ; \mathrm{p}=.011)$. Es decir, eligen la televisión como actividad de ocio en una cuantía significativamente menor que la media del resto de jóvenes.

Tabla 3 . Medias (M), desviaciones típicas (DT), resultados de la prueba de contrastes de medias y significación con respecto a los valores y escuchar música.

\begin{tabular}{|c|c|c|c|c|c|c|c|c|}
\hline & \multicolumn{2}{|l|}{ Total } & \multicolumn{2}{|c|}{$\begin{array}{l}\text { Escuchan } \\
\text { música } \\
(\mathbf{N}=356)\end{array}$} & \multicolumn{2}{|c|}{$\begin{array}{l}\text { No escuchan } \\
\text { música } \\
(\mathrm{N}=25)\end{array}$} & \multirow[b]{2}{*}{$\begin{array}{l}\text { Prueba } \\
\mathbf{t}\end{array}$} & \multirow[b]{2}{*}{ Sig. (bilat.) } \\
\hline & $\mathbf{M}$ & DT & $\mathbf{M}$ & DT & $\mathbf{M}$ & DT & & \\
\hline Benevolencia & 7.236 & 1.022 & 7.227 & 1.019 & 7.392 & 1.097 & -.776 & .438 \\
\hline Seguridad & 7.024 & .968 & 7.025 & .973 & 6.944 & .940 & .407 & .684 \\
\hline Universalismo & 6.623 & 1.071 & 6.651 & 1.060 & 6.350 & 1.073 & 1.371 & .171 \\
\hline Conformidad & 7.116 & 1.160 & 7.107 & 1.169 & 7.290 & 1.017 & -.759 & .448 \\
\hline Tradición & 5.819 & 1.086 & 5.831 & 1.084 & 5.672 & 1.050 & .712 & .477 \\
\hline Logro & 6.786 & 1.146 & 6.786 & 1.130 & 6.850 & 1.233 & -.270 & .787 \\
\hline Poder & 5.103 & 1.388 & 5.106 & 1.365 & 4.940 & 1.373 & .589 & .556 \\
\hline Hedonismo & 7.148 & 1.285 & 7.175 & 1.278 & 6.760 & 1.392 & 1.560 & .120 \\
\hline Estimulación & 6.480 & 1.388 & 6.531 & 1.370 & 6.531 & 1.370 & 2.657 & $.008 *$ \\
\hline Autodirección & 7.217 & .930 & 7.251 & .892 & 7.251 & .892 & 2.739 & $.006^{*}$ \\
\hline
\end{tabular}

Fuente: Elaboración propia. ${ }^{*} \rho<.05$. Se han asumido varianzas iguales.

Por último, en referencia al disfrute de la música como actividad de ocio, los jóvenes que se identifican con los valores estimulación $(\mathrm{t}=2.657 ; \mathrm{p}=.008)$ y autodirección $(\mathrm{t}=2.739$; 
$\mathrm{p}=.006)$ son los que obtienen medias estadísticamente más altas que el resto del grupo.

Ambos valores están representados cercanos en el modelo gráfico de Schwartz y están englobados en el valor de orden superior Apertura al cambio, lo que permite identificar un perfil axiológico concreto que muestra especial preferencia por escuchar música en su tiempo libre.

Con todo, observando las cinco actividades de ocio en la que se emplean tecnología de la información y la comunicación, se observa que los valores estimulación, autodirección y seguridad obtienen valores estadísticamente significativos en el contraste entre los diferentes perfiles axiológicos. Estos tres valores se agrupan en los valores de orden superior Apertura al cambio -estimulación y autodirección- y Conservadurismo -seguridad- y éstos, a su vez, enfrentados entre sí, lo que permite establecer perfiles axiológicos según la preferencia de un tipo u otro de actividad de ocio relacionada con las TIC.

Por otro lado, si analizamos la preferencia de las diferentes actividades de ocio en función del sexo de los participantes, los resultados evidencian, mediante las pruebas Chicuadrado, Phi y V de Cramer que no existe asociación significativa $(\chi 2=.105 ; \mathrm{p}=.746$; Phi=.017, $\mathrm{p}=.001 ; \mathrm{V}$ de Cramer=.017, $\mathrm{p}=.746$ ) en el modo en que los chicos y las chicas ven la TV. Si bien, se observa que ambos sexos afirman que ven la TV en un $85.1 \%$ (véase Tabla $4)$.

Tabla 4. Distribución de participantes para la actividad Ver TV según su sexo. Tabla de contingencia con Prueba $\chi 2$ Ver TV.

\begin{tabular}{llll}
\hline \multirow{2}{*}{ Ver TV } & \multicolumn{3}{c}{ Sexo } \\
\cline { 2 - 4 } & Varón & Mujer & N \\
\hline \multirow{2}{*}{ Sí } & 162 & 164 & 326 \\
& $85.7 \%$ & $84.5 \%$ & $85.1 \%$ \\
\hline \multirow{2}{*}{ No } & 27 & 30 & 57 \\
& $14.3 \%$ & $15.5 \%$ & $14.9 \%$ \\
\multirow{2}{*}{ Total } & 189 & 194 & 383 \\
& $100 \%$ & $100 \%$ & $100 \%$ \\
\hline
\end{tabular}

Fuente: Elaboración propia.. Prueba Chi-cuadrado $(\chi 2)=.105$, Grados de Libertad $(\mathrm{gl})=1$, Probabilidad de significación $(\mathrm{p})=.746$. Prueba Phi $=.017$.

Probabilidad de significación $(\mathrm{p})=.746$. Prueba $\mathrm{V}$ de Cramer $=.017$. Probabilidad de significación $(p)=.746$.

Algo parecido ocurre con la variable escuchar la radio. Como se puede apreciar en la Tabla 5, los resultados hallados determinan que no existe asociación significativa $(\chi 2=1.746$; 
$\mathrm{p}=.186 ; \mathrm{Phi}=.068, \mathrm{p}=.186 ; \mathrm{V}$ de Cramer=.068, $\mathrm{p}=.186$ ) en el modo en que los chicos y las chicas escuchan la radio. En este caso, los porcentajes disminuyen respecto a ver la TV, pues únicamente un $52.6 \%$ del total de la muestra afirma que escucha la radio.

Tabla 5, Distribución de participantes para la actividad Escuchar radio según su sexo.. Tabla de contingencia con Prueba $\chi 2$ Escuchar radio.

\begin{tabular}{llll}
\hline \multirow{2}{*}{ Escuchar radio } & \multicolumn{3}{c}{ Sexo } \\
\cline { 2 - 4 } & Varón & Mujer & N \\
\hline \multirow{2}{*}{ Sí } & 93 & 108 & 201 \\
& $49.2 \%$ & $56.0 \%$ & $52.6 \%$ \\
\hline \multirow{2}{*}{ No } & 96 & 85 & 181 \\
\multirow{2}{*}{ Total } & $25.1 \%$ & $22.3 \%$ & $47.4 \%$ \\
& 189 & 193 & 382 \\
& $100 \%$ & $100 \%$ & $100 \%$ \\
\hline
\end{tabular}

Fuente: Elaboración propia.. Prueba Chi-cuadrado $(\chi 2)=1.746$, Grados de Libertad $(\mathrm{gl})=1$, Probabilidad de significación $(\mathrm{p})=.186$. Prueba Phi $=-.068$.

Probabilidad de significación $(\mathrm{p})=.186$. Prueba $\mathrm{V}$ de Cramer $=.068$. Probabilidad de significación $(\mathrm{p})=.186$.

En la línea de los anteriores resultados, tampoco se encuentra ninguna asociación significativa entre el sexo y la utilización del $P C$ (véase Tabla 6. $\chi 2=.196 ; \mathrm{p}=.658 ; \mathrm{Phi}=.023$, $\mathrm{p}=.658 ; \mathrm{V}$ de Cramer=.068, p=.658). La utilización del $P C$ es generalizada para ambos sexos, dado que el $83.8 \%$ de la muestra afirma que lo utiliza.

Tabla 6. Distribución de participantes según su sexo. Tabla de contingencia con Prueba $\chi 2$ Utilizar el PC.

\begin{tabular}{llll}
\hline \multirow{2}{*}{ Utilizar el PC } & \multicolumn{3}{c}{ Sexo } \\
\cline { 2 - 4 } & Varón & Mujer & N \\
\hline \multirow{2}{*}{ Sí } & 160 & 161 & 321 \\
& $84.7 \%$ & $83.0 \%$ & $83.8 \%$ \\
\hline \multirow{2}{*}{ No } & 29 & 33 & 62 \\
& $15.3 \%$ & $17 \%$ & $16.2 \%$ \\
\multirow{2}{*}{ Total } & 189 & 194 & 383 \\
& $100 \%$ & $100 \%$ & $100 \%$ \\
\hline
\end{tabular}

Fuente: Elaboración propia.. Prueba Chi-cuadrado $(\chi 2)=.196$, Grados de Libertad $(\mathrm{gl})=1$, Probabilidad de significación $(\mathrm{p})=.658$. Prueba Phi $=.023$.

Probabilidad de significación $(\mathrm{p})=.658$. Prueba $\mathrm{V}$ de Cramer $=.023$. Probabilidad de significación $(p)=.658$. 
Finalmente, en el caso de jugar a videojuegos y su relación con el sexo de los participantes, en la Tabla 7 se muestran resultados que indican que sí existe una asociación significativa alta $(\chi 2=.121 .216 ; \mathrm{p}=.000 ; \mathrm{Phi}=.563, \mathrm{p}=.000 ; \mathrm{V}$ de Cramer=.563, $\mathrm{p}=.000)$. De tal manera, se observa que existe un mayor porcentaje de jóvenes varones que afirman jugar a videojuegos (69.3\%) frente a las mujeres (13.9\%). De tal manera, si consideramos acumular, por un lado, los porcentajes de aquellas respuestas que contemplan que sí juegan a videojuegos y, por otro lado, aquellos que no juegan a videojuegos, apreciamos que los jóvenes afirman que juegan a videojuegos en un $41.3 \%$ frente un $58.7 \%$ que afirma que no juega en su tiempo de ocio.

Tabla 7. Distribución de participantes según su sexo. Tabla de contingencia con Prueba $\chi^{2}$ Jugar a videojuegos.

\begin{tabular}{llll}
\hline \multirow{2}{*}{$\begin{array}{c}\text { Jugar a } \\
\text { videojuegos }\end{array}$} & Varón & Mujer & N \\
\cline { 3 - 4 } Sí & 131 & 27 & 158 \\
& $69.3 \%$ & $13.9 \%$ & $41.3 \%$ \\
\hline \multirow{2}{*}{ No } & 58 & 167 & 225 \\
& $30.7 \%$ & $86.1 \%$ & $58.7 \%$ \\
\multirow{2}{*}{ Total } & 189 & 194 & 383 \\
& $100 \%$ & $100 \%$ & $100 \%$ \\
\hline
\end{tabular}

Fuente: Elaboración propia. Prueba Chi-cuadrado $(\chi 2)=121.216$, Grados de Libertad $(\mathrm{gl})=1$, Probabilidad de significación $(p)=.000$. Prueba Phi $=.563$.

Probabilidad de significación $(\mathrm{p})=.000$. Prueba $\mathrm{V}$ de Cramer $=.563$. Probabilidad de significación $(\mathrm{p})=.000$.

Por último, en la Tabla 8, analizando la diferencia del uso de los recursos tecnológicos en el tiempo de ocio de los jóvenes en función de los grupos de edad; oscilando éstos entre los 13 y los 19 años, podemos ver que no existen diferencias estadísticamente significativas en ninguna de las actividades. Es decir, todos los jóvenes, independientemente de su edad, emplean las TIC de manera semejantes, sin que el proceso evolutivo marque ninguna diferencia.

Tabla 8. Análisis de varianza de las preferencias de utilización de los recursos tecnológicos en el tiempo libre en función de la edad.

$\begin{array}{llllll}\text { Suma } & \text { de } & \text { gl } & \text { Media } & \text { F } & \text { Sig. }\end{array}$




\begin{tabular}{|c|c|c|c|c|c|c|}
\hline & & cuadrad & & $\begin{array}{l}\text { cuadrátic } \\
\text { a }\end{array}$ & & \\
\hline \multirow{3}{*}{ Ver TV } & Entre & 1.482 & 6 & .247 & 1.996 & .065 \\
\hline & Intra & 46.287 & 374 & .124 & & \\
\hline & Total & 47.769 & 380 & & & \\
\hline \multirow{3}{*}{ Oir radio } & Entre & 1.650 & 6 & .275 & 1.102 & .360 \\
\hline & Intra & 93.087 & 373 & .250 & & \\
\hline & Total & 94.737 & 379 & & & \\
\hline \multirow{3}{*}{ Usar PC } & Entre & .705 & 6 & .117 & .870 & .517 \\
\hline & Intra & 50.529 & 374 & .135 & & \\
\hline & Total & 51.234 & 380 & & & \\
\hline \multirow{3}{*}{$\begin{array}{l}\text { Jugar } \\
\text { videojuegos }\end{array}$} & Entre & 2.212 & 6 & .369 & 1.530 & .167 \\
\hline & Intra & 90.092 & 374 & .241 & & \\
\hline & Total & 92.304 & 380 & & & \\
\hline \multirow{3}{*}{$\begin{array}{l}\text { Escuchar } \\
\text { música }\end{array}$} & Entre & .440 & 6 & .073 & 1.198 & .306 \\
\hline & Intra & 22.919 & 374 & .061 & & \\
\hline & Total & 23.360 & 380 & & & \\
\hline
\end{tabular}

\section{Discusión y conclusiones}

Los resultados obtenidos permiten concluir la existencia de una relación entre los valores de los jóvenes y el uso de TIC en su tiempo de ocio al encontrar, al menos, seis valores que obtienen relación estadísticamente significativa con algunas de las actividades analizadas. No obstante, esta relación muestra diferencias estadísticamente significativas que permiten clasificar la utilización que hacen los jóvenes de las TIC en función de los valores que definen su sistema axiológico, distribuyéndose en tres de los cuatro valores de orden superior y pudiéndose definir varios perfiles axiológicos según la actividad de tiempo de ocio realizada.

De este modo, los jóvenes caracterizados por el valor de orden superior Apertura al cambio -hedonismo, estimulación y autodirección- suelen destacar por la utilización de tecnologías más relacionadas con el entretenimiento -videojuegos y música- y con la información -TV-. Esto resulta coherente con el contenido motivacional expuesto por Schwartz para los jóvenes identificados con estos valores, que buscan controlar el medio, así como la búsqueda de situaciones y actividades estimulantes que provean de vivencias y sensaciones fuertes (Torres, Schwartz, \& Nascimento, 2016). No obstante, estos datos contrastan con los resultados encontrados por Martínez (2015), que afirman que los videojuegos transmiten tanto valores individualistas, característicos de los perfiles de Apertura al cambio y Auto-promoción, como colectivistas, propios de los valores de conservadurismo y auto-trascendencia. Similares conclusiones apuntan Ortega y Robles 
(2008); Medrano, Aierbe y Martínez, (2011); y Sinde, Medrano y Ignacio (2015).

De igual manera, los jóvenes identificados con el valor poder destacan también por la preferencia de los videojuegos como actividad de ocio, siendo las características de este tipo de entretenimiento coincidente con la necesidad de dominio y control característico de este axioma.

Por otro lado, el valor de orden superior conservación, que integra los valores de seguridad, conformidad y tradición, destaca por la preferencia de la radio y el rechazo del PC como actividad de ocio. Aun sin conocer el contenido preferido para ambos medios, es significativa la preferencia de la radio como tecnología unidireccional y que no permite la interacción del receptor y, por otro lado, el rechazo del PC, como recurso tecnológico que favorece la interacción con otras personas a través de plataformas digitales. Esto es coherente con las actitudes descritas por Schwartz para estos valores, que rechazan las conductas individuales potencialmente disruptivas para el funcionamiento armónico del grupo.

Además, cabe destacar que ninguno de los valores pertenecientes al valor de orden superior auto-trascencencia -benevolencia y universalismo- difieren significativamente del resto de perfiles axiológicos en el uso de recursos tecnológicos en su tiempo libre. Esto no significa que no utilicen las TIC, sino que no hacen una utilización significativamente mayor o menor de las mismas. Estos resultados son coherentes con la fundamentación motivacional de ambos valores, centrados en la búsqueda del bien común y con actitudes y conductas proclives al voluntariado, la entrega a los demás y la defensa de los derechos sociales, tal y como exponen De la Fuente, Martínez y Chaparro (2003) y Tobón, Zapata, Lopera y Duque (2014).

Por otro lado, es importante observar los análisis de la diferencia en la preferencia de utilización de las TIC en el tiempo de ocio de los jóvenes en función de sus características sociodemográficas. Así, si se clasifican los jóvenes en función del sexo se observa que no existen diferencias estadísticamente significativas en el utilización de ninguno de los recursos tecnológicos, salvo en el de los videojuegos, donde los hombres destacan por una mayor preferencia que las mujeres. De nuevo, estos datos son totalmente coincidentes con trabajos previos llevados a cabo por Protégeles, Civertice y Defensor del Menor (2005) o Martínez (2015), que también encuentran que el índice de utilización de los videojuegos es mayor en los hombres.

Sin embargo, se observa que no hay diferencias significativas entre los grupos de 
edad, mostrando una preferencia de utilización pareja de los recursos tecnológicos en el tiempo de ocio. Estos resultados son coherentes con los encontrados por Martínez (2015) que, aunque limita su investigación al uso de videojuegos, mantiene que la edad no es un factor determinante para justificar las diferencias encontradas entre los distintos grupos formados por este criterio.

Con todo, la clasificación de las diferencias de utilización de los recursos tecnológicos en el tiempo de ocio de los jóvenes en función de los valores, permite hacer un diseño más ajustado de los programas educativos, respondiendo con mayor concreción a las demandas e inquietudes de cada perfil axiológico.

Por otro lado, la presente investigación abre la puerta a estudiar el contenido concreto de la utilización de las TIC que hacen los jóvenes según su perfil axiológico. Así, es importante diferenciar el uso lúdico de la televisión del uso informativo. Algo similar ocurre con la radio y el PC, cuyo uso puede diferir significativamente entre lúdico, informativo y comunicativo.

Por último, la utilización cada vez más creciente del móvil a edades más tempranas ([Autor/a]) hace necesario ampliar la presente investigación incluyendo este recurso tecnológico como actividad de ocio y ver las diferencias de uso según el sistema de valores de los jóvenes.

\section{REFERENCIAS BIBLIOGRÁFICAS}

Amorós, P., Buxarrais, M. R. \& Casas, F. (2002). La influencia de las TIC en la vida de los chicos y chicas de 12 a 16 años. Barcelona: Instituto de Infancia y Mundo Urbano.

Asociación Médica Mundial (AMM) (2013). Declaración de Helsinki de la AMM - Principios éticos para las investigaciones médicas en seres humanos. Recuperado de http://www.wma.net/es/30publications/10policies/b3http://www.wma.net/es/30publications/10policies $/ \mathrm{b} 3$

Corvalán, J. (2012). El ocio y tiempo libre en la sociedad actual. Contribuciones a las Ciencias Sociales, (2012-07). Recuperado de http://www.eumed.net/rev/cccss/21/jlmc.pdf

De la Fuente Robles, Y., Martínez, J. \& Chaparro, M. P. M. (2003). Los valores personales y la participación de estudiantes de Psicopedagogía en organizaciones de voluntariado. Revista de psicología social aplicada, 13(3), 61-90.

Del Barrio, Martínez (2016). Los abuelos y su influencia en el uso del tiempo libre y las actividades extraescolares de los nietos jóvenes. International Journal of Developmental and Educational Psychology. Revista INFAD de Psicología., 1(2), 207-218. 
Delgado, M., Arrieta, X. \& Riveros, V. (2009). Uso de las TIC en educación, una propuesta para su optimización. Omnia, 15(3), 58-77.

Elizalde, L. (2016). Los jóvenes y la tecnologías de la comunicación y de la información. Hacia una etnografía de los entornos mediáticos. ZER-Revista de Estudios de Comunicación, 3(5), 121-156.

Fernández-García, A., Poza-Vilches, F. \& Fiorucci, M. (2015). Análisis metateórico sobre el ocio de la juventud con problemas sociales. Pedagogía Social. Revista Interuniversitaria, 25, 25-49.

Ferreira, P., Pose, H. \& De Valenzuela, A. (2015). El ocio cotidiano de los estudiantes de Educación Secundaria en España. Pedagogía Social. Revista Interuniversitaria, 25, 119-142.

García, V., Barbero, F. \& Muñoz, R. (2017). Evaluación de la jerarquía de los valores humanos de Schwartz en la adolescencia: diferencias de género e implicaciones educativas. Revista Brasileira de Educação, 22(68), 123-146.

González, M., Fernández, M. E. \& Urturi, A. (2015). Uso y riesgos de las tecnologías de la información y comunicación en adolescentes de 13-18 años. Acta Pediátrica Española, 73(6), 126135 .

Loader, B. D., Vromen, A. \& Xenos, M. A., (2014). The networked young citizen: social media, political participation and civic engagement. Information, Communication \& Society, 17(2), 143-150.

Lujambio, L. U., Martínez, J. I. \& Samaniego, M. C. M. (2017). Pautas de consumo televisivo en adolescentes de la era digital: un estudio transcultural. Comunicar: Revista científica iberoamericana de comunicación y educación, 25(50), 67-76.

Matza, D. \& Sykes, G. (2014). Delincuencia juvenil y valores subterráneos. Delito y sociedad, 23(38), 119-129.

Medrano, C., Aierbe, A. \& Martínez J. I. (2011): Valores percibidos en el medio televisivo por jóvenes en contextos transculturales. Comunicar, 19(37), 117-124.

Medrano \& Aierbe (2008). Valores y contextos de desarrollo. Revista de Psicodidáctica, 13(1), 53-68.

Melendro, M., García-Castilla, F. J. \& Goig, R. (2016). El uso de las TIC en el ocio y la formación de los jóvenes vulnerables. Revista Española de Pedagogía, 74 (218), 71-89.

Montero, Y. (2013). Televisión, valores y jóvenes. Barcelona: Gedisa.

Ortega, J. \& Robles, M. (2008): “Análisis axiológico-educativo de videojuegos de temática violenta". Revista Electrónica Teoría de la Educación: Educación y Cultura en la Sociedad de la Información, 9(3), 150-166.

Ortega, C., Lazcano, I. \& Rocha, M. (2015). Espacios de ocio para jóvenes, de la monitorización a la autogestión. Pedagogía Social. Revista Interuniversitaria, 25, 69-90.

Solano, I. M., González, V. \& López, P. (2013). Jóvenes y comunicación: las TIC como recurso para la interacción social en educación secundaria. Pixel-Bit. Revista de Medios y Educación, 2013(42), 23 35 .

Samaniego, C, Cortés, A. \& Navarro, S. (2009). Los valores personas y los valores percibidos en la 
televisión: un estudio con jóvenes. Revista electrónica interuniversitaria de formación del profesorado, 12(4), 55-66.

Sinde, J., Medrano, C. \& Ignacio, J. (2015): Transmisión de valores en jóvenes: un análisis con videojuegos. Revista Latina de Comunicación Social, 70, 230-251.

Tobón, O., Zapata, S., Lopera, I. \& Duque, J. (2014). Formación académica, valores, empatía y comportamientos socialmente responsables en estudiantes universitarios. Revista de la educación superior, 43(169), 89-105.

Torres, C., Schwartz, S. \& Nascimento, T. (2016). A Teoria de Valores Refinada: associações com comportamento e evidências de validade discriminante e preditiva. Psicologia USP, 27(2), 341-356. Van Quaquebeke, N., Graf, M. M., Kerschreiter, R., Schuh, S. C. \& Dick, R. (2014). Ideal Values and Counter-ideal Values as Two Distinct Forces: Exploring a Gap in Organizational Value Research. International Journal of Management Reviews, 16(2), 211-225.

Wieckowski, A. T. \& White, S. W. (2017). Application of technology to social communication impairment in childhood and adolescence. Neuroscience \& Biobehavioral Reviews, 74(A), 98-114.

Zambrano, E. Y. C. \& Badillo, C. S. H. (2016). El aislamiento social como consecuencia del uso excesivo de internet y móviles en adolescentes. PsicoEducativa: reflexiones y propuestas, 2(4), 16-22.

\section{Original recebido em: 23 de outubro de 2017}

Aceito para publicação em: 30 de novembro de 2017

Javier Páez Gallego

Profesor del Máster de Intervención Educativa en Contextos Sociales (UNED). Doctor en Ciencias de la Educación por la UNED. Centra su interés en la educación en valores.

Angel De-Juanas Oliva Ángel De-Juanas Oliva es profesor del Departamento de Teoría de la Educación y Pedagogía Social de la UNED. Doctor en Ciencias de la Educación por la UCM. Investigador del Grupo de Intervención Socioeducativa.

Ángel Luis Gonzáles Olivares

Profesor Asociado. Departamento de Pedagogía. Facultad de Educación de Ciudad Real. Universidad de Castilla La Mancha.

Esta obra está licenciada sob uma Licença Creative Commons. 